\title{
Cardiomyocyte-derived adiponectin is biologically active in protecting against myocardial ischemia-reperfusion injury.
}

\author{
Yajing Wang \\ Thomas Jefferson University \\ Wayne Bond Lau \\ Thomas Jefferson University \\ Erhe Gao \\ Thomas Jefferson University \\ Ling Tao \\ Xijing Hospital \\ Fulfoxinthisuand additional works at: https://jdc.jefferson.edu/emfp \\ Thomas Jefferson University \\ Part of the Emergency Medicine Commons

\section{Let us know how access to this document benefits you See next page for additional authors}

\section{Recommended Citation}

Wang, Yajing; Lau, Wayne Bond; Gao, Erhe; Tao, Ling; Yuan, Yuexing; Li, Rong; Wang, Xiaoliang; Koch, Walter J.; and Ma, Xin-Liang, "Cardiomyocyte-derived adiponectin is biologically active in protecting against myocardial ischemia-reperfusion injury." (2010). Department of Emergency Medicine Faculty Papers. Paper 157.

https://jdc.jefferson.edu/emfp/157

This Article is brought to you for free and open access by the Jefferson Digital Commons. The Jefferson Digital Commons is a service of Thomas Jefferson University's Center for Teaching and Learning (CTL). The Commons is a showcase for Jefferson books and journals, peer-reviewed scholarly publications, unique historical collections from the University archives, and teaching tools. The Jefferson Digital Commons allows researchers and interested readers anywhere in the world to learn about and keep up to date with Jefferson scholarship. This article has been accepted for inclusion in Department of Emergency Medicine Faculty Papers by an authorized administrator of the Jefferson Digital Commons. For more information, please contact: JeffersonDigitalCommons@jefferson.edu. 


\section{Authors}

Yajing Wang, Wayne Bond Lau, Erhe Gao, Ling Tao, Yuexing Yuan, Rong Li, Xiaoliang Wang, Walter J. Koch, and Xin-Liang Ma 
2 Cardiomyocyte-Derived Adiponectin is Biologically Active in Protecting Against Myocardial Ischemia/Reperfusion Injury

5 Yajing Wang, MD, $\mathrm{PhD}^{1}$, Wayne Bond Lau, $\mathrm{MD}^{1}$, Erhe Gao, MD, $\mathrm{PhD}^{2}$, Ling Tao, $\mathrm{MD}, \mathrm{PhD}^{3}$, Yuexin Yuan, $\mathrm{PhD}^{1}$, Rong Li, MD, $\mathrm{PhD}^{1}$, Xiaoliang Wang, $\mathrm{MD}, \mathrm{PhD}$, Walter Koch, $\mathrm{PhD}^{2}$, Xin-Liang $\mathrm{Ma}, \mathrm{MD}, \mathrm{PhD}^{1 \#}$

Running Title: Cardiac Adiponectin and Myocardial Protection

12 Address proofs to:

$13{ }^{\text {\#} X i n ~ L ~ M a, ~ M . D ., ~ P h . D . ~}$

14 Department of Emergency Medicine

151020 Sansom Street

16 Thompson Building, Room 239

17 Philadelphia, PA 19107

18

19 Tel: (215)955-4994

20 Fax: (215)923-6225

21 E-mail: Xin.Ma@Jefferson.edu

22

23

24 Total Word Count: 4,971

25

$26{ }^{1}$ Department of Emergency Medicine, ${ }^{2}$ Center for Translational Medicine, Thomas Jefferson University,

27 Philadelphia, PA 19107, ${ }^{3}$ Department of Cardiology, Xijing Hospital, 15 West Changle Road, Xian, PR

28 China. 
30 Adiponectin (APN) has traditionally been viewed as an adipocyte-specific endocrine molecule

31 with cardioprotective effects. Recent studies suggest that APN is also expressed in

32 cardiomyocytes. However, biological significances of this locally produced APN remain

33 completely unknown. The aim of this study was to investigate the pathologic and pharmacologic

34 significance of cardiac-derived APN in cardiomyocyte pathology. Adult cardiomyocytes from

35 wild type littermates (WT) or gene deficient mice were pre-treated with vehicle (V) or

36 rosiglitazone (RSG) for $6 \mathrm{~h}$ followed by simulated ischemia/reperfusion (SI/R, 3h/12h).

37 Compared to WT cardiomyocytes, myocytes from APN knockout (APN-KO) mice sustained

38 greater SI/R injury, evidenced by greater oxidative/nitrative stress, caspase-3 activity, and LDH

39 release $(\mathrm{P}<0.05)$. Myocytes from adiponectin receptor 1 knock-down (AdipoR1-KD) or

40 AdipoR1-KD/AdipoR2-KO mice had slightly increased SI/R injury, but the difference was not

41 statistically significant. RSG significantly $(\mathrm{P}<0.01)$ increased APN mRNA and protein

42 expression, upregulated AdipoR1/AdipoR2 expression, reduced SI/R-induced apoptosis, and

43 decreased LDH release in WT cardiomyocytes. However, the anti-oxidative/anti-nitrative and

44 cell protective effects of RSG were completely lost in APN-KO cardiomyocytes ( $\mathrm{P}>0.05$ vs.

45 vehicle group), although a comparable degree of AdipoR1/AdipoR2 upregulation was observed.

46 The upregulatory effect of RSG on APN mRNA and protein expression was significantly

47 potentiated in AdipoR1-KD/AdipoR2-KO cardiomyocytes. However, the cellular protective

48 effects of RSG were significantly blunted, although not completely lost, in these cells. These

49 results demonstrated that cardiomyocyte APN is biologically active in protecting cells against

$50 \mathrm{SI} / \mathrm{R}$ injury. Moreover, this locally produced APN achieves its protective effect primarily

51 through paracrine/autocrine activation of APN receptors. 
52 Keywords: Myocardial ischemia; diabetes; cytokines; oxidative stress 
Strong epidemiological evidence exists that type-2 diabetes not only causes coronary

55 vascular injury thus increasing ischemic heart disease prevalence in these patients, but also

56 renders cardiomyocytes more susceptible to ischemia/reperfusion insult, and increases

57 cardiomyocyte death after the onset of myocardial ischemia( $5 ; 15 ; 19)$. Clarifying the molecular

58 link between type-2 diabetes and cardiovascular injury may therefore help identify novel

59 effective therapeutic interventions that attenuate post-ischemic myocardial injury, reduce

60 myocardial ischemic morbidity, and ultimately decrease diabetic mortality of cardiovascular

61 etiology.

62 Adiponectin (APN) is a protein hormone circulating in plasma as multimeric complexes

63 at relatively high concentration $(2-10 \mu \mathrm{g} / \mathrm{ml})(21)$. Besides its well-defined insulin sensitization

64 and metabolic regulatory effects, recent experimental and clinical studies demonstrate that APN

65 is a potent endogenous cardioprotective molecule(10). Numerous epidemiological studies have

66 shown that reduced APN levels correlate with increased risk of cardiovascular disease in obesity

67 and diabetes $(9 ; 12 ; 16 ; 29)$; high plasma APN concentrations are associated with a lower risk of

68 MI in men(23). In addition, recent clinical observations have demonstrated that post-MI plasma

69 APN levels correlated positively with myocardial salvage index and ejection fraction

70 recovery(24). Persistently low plasma APN concentrations after acute myocardial infarction are

71 predictive of future adverse cardiac events(1). As such, reduced APN production has been

72 recognized as a risk factor for cardiovascular disease, and enhancing APN production has been

73 accepted as a potential therapeutic modality for ameliorating diabetic cardiovascular injury.

74 Although APN receptors, including APN receptor 1 (AdipoR1) and APN receptor 2

75 (AdipoR2), are present in most organs including adult cardiomyocytes, it was generally accepted

76 until recently that APN is exclusively synthesized in adipocytes. However, new studies reveal 
77 that the APN gene is expressed in other cell types, including hepatocytes, myotubes, skeletal 78 muscle, and osteoblasts $(17 ; 25)$. Three recent studies have demonstrated that APN is also 79 expressed in adult cardiomyocytes, and its production is increased by activation of peroxisome 80 proliferator-activated receptor- $\gamma(\operatorname{PPAR} \gamma)(4 ; 11 ; 22)$. However, whether this cardiac-derived 81 APN is biologically active, contributing to physiological and pathologic regulation of 82 cardiomyocyte function, remains completely unknown presently. Addressing this critical 83 question may provide clues to the largely unknown regulation of cardiomyocyte metabolism and 84 function in the diabetic heart.

85 Therefore, the aims of the present study were to determine whether cardiomyocyte86 derived APN is biologically active and cardioprotective, and if so, to further investigate 87 transmembrane and intracellular signaling mechanisms responsible for the cardioprotective 88 actions of locally produced APN. 
Materials and Methods:

97 Animals: Adult male C57BL/6, homozygous adiponectin knockout (APN-KO), AdipoR2

98 homozygous knockout (AdipoR2-KO), and their littermate wild type control weighing 27-32 g

99 were purchased from Jackson Labs, Inc (Bar Harbor, ME). All procedures were performed in 100 accordance with the National Institutes of Health Guidelines on the Use of Laboratory Animals, 101 and were approved by the Thomas Jefferson University Committee on Animal Care.

102 In vivo siRNA-mediated AdipoR1 knock-down (AdipoR1-KD): AdipoR1 knockout mice were not

103 commercially available when this study was performed. We thus utilized siRNA gene silencing

104 technique to knock-down AdipoR1 expression in mouse heart (AdipoR1-KD). In brief, three pre105 designed AdipoR1-specific siRNA (catalog numbers: s91209, s91210, and s91208, Ambion 106 Silencer) or a control nonspecific siRNA oligos (Silencer Select Negative Control \#1 siRNA, 107 Ambion) were diluted in 5\% glucose and mixed with in vivo-jet PEI (Genesee Scientific, San 108 Diego, CA). Adult wild type (WT) or AdipoR2-KO mice were anesthetized with 2\% isoflurane, 109 and the heart was exposed via left thoracotomy at the fifth intercostal space. $20 \mu 1(0.8 \mu \mathrm{g} / \mathrm{g})$ 110 siRNA or negative control was delivered via three separate intramyocardial injections (32 $1 / 2 \mathrm{G}$ 111 needle) to temporarily blanch the left ventricular free wall. Based on our pilot experiments 112 showing that AdipoR1 expression reaches its nadir $48 \mathrm{~h}$ after siRNA injection, hearts were 113 isolated $48 \mathrm{~h}$ following siRNA treatment for adult cardiomyocyte harvest and preparation as 114 described below.

115 Preparation and culturing of adult mouse cardiomyocytes: Mice were anesthetized with 2\% 116 isoflurane. Hearts were removed and perfused at $37^{\circ} \mathrm{C}$ for $\sim 3$ min with a $\mathrm{Ca}^{2+}$-free bicarbonate117 based buffer. Enzymatic digestion was initiated by adding collagenase type B/D to the perfusion 118 solution. After $\sim 3$ min of digestion, at which point the cardiac tissue became firm and swollen, 
$11950 \mu \mathrm{M} \mathrm{Ca}^{2+}$ was added to the enzyme solution; $\sim 7$ min later, the left ventricle was removed, cut

120 into several sections, and further digested in a shaker for $10 \mathrm{~min}$ at $37^{\circ} \mathrm{C}$ in the same enzyme

121 solution. The supernatant containing the dispersed myocytes was filtered into a sterilized tube,

122 and centrifuged at $800 \mathrm{x}$ g for $1 \mathrm{~min}$. The cell pellet was then resuspended in bicarbonate-based

123 buffer containing $125 \mu \mathrm{M} \mathrm{Ca}^{2+}$. After the myocytes were pelleted by gravity for $\sim 10 \mathrm{~min}$, the

124 supernatant was aspirated, and the myocytes were resuspended in bicarbonate-based buffer 125 containing $250 \mu \mathrm{M} \mathrm{Ca}^{2+}$. Myocytes were plated at $0.5-1 \times 10^{4}$ cells. $\mathrm{cm}^{-2}$ in culture dishes pre126 coated with mouse laminin.

127 Simulated ischemia/reperfusion (SI/R). After $1 \mathrm{~h}$ of culture in a $5 \% \mathrm{CO}_{2}$ incubator at $37^{\circ}$ $128 \mathrm{C}$, cardiomyocytes were randomized to receive either vehicle or rosiglitazone treatment (RSG, $12910 \mu \mathrm{mol} / \mathrm{L})(4)$. Six h after RSG or vehicle treatment, cells were either collected for assessment of $130 \mathrm{APN}$ and its receptor expression, or further subjected to simulated ischemia/reperfusion (SI/R) as 131 originally described by Isner and colleagues(20) and modified in our recently published study(8).

132 In brief, glucose-free culture medium was first gassed for 5 minutes with a hypoxic gas mixture $133\left(95 \% \mathrm{~N}_{2} / 5 \% \mathrm{CO}_{2}\right)$. Normal culture medium was quickly replaced with the hypoxia-hypoglycemic 134 medium, and cardiomyocytes were placed in a Napco $8000 \mathrm{WJ}$ hypoxia $\left(1 \% \mathrm{O}_{2} / 5 \% \mathrm{CO}_{2} / 94 \% \mathrm{~N}_{2}\right)$ 135 incubator (Thermo Scientific, Waltham, MA). After $3 \mathrm{~h}$ of hypoxia-hypoglycemic culture, the 136 hypoxia/hypoglycemic medium was replaced with normal culture medium. Cells were then 137 incubated for an additional $12 \mathrm{~h}$ under normoxic conditions in a $\mathrm{CO}_{2}$ incubator.

138 Measurements of mRNA levels in cultured cardiomyocytes: Total RNA was extracted from 139 cultured cardiomyocytes with a Qiagen RNeasy kit (Qiagen, Valencia, CA). The expression 140 levels of mRNA for APN, AdipoR1, and AdipoR2 were quantified by TaqMan one Step RT141 PCR Master Mix reagent kit (Applied Biosystems, Foster City, CA) using FAM-labeled Taqman 
142 probes (APN, AdipoR1, AdipoR2, Applied Biosystems) and $100 \mathrm{ng}$ sample RNA to a final

143 volume of $10 \mu \mathrm{l}$. Amplification reactions were performed in ABI 7900HT Sequence Detection

144 System (Applied Biosystems) in the 384-well block format with the following cycle conditions:

$14550^{\circ} \mathrm{C}$ for 20 min for reverse transcription, $95^{\circ} \mathrm{C}$ denaturation and Ampli Taq Gold DNA

146 polymerase activation for $10 \mathrm{~min}$, followed by 40 cycles of $95^{\circ} \mathrm{C}$ denaturation for $15 \mathrm{~s}$, and $60^{\circ} \mathrm{C}$

147 annealing and elongation for $60 \mathrm{~s}$. Fluorescence spectra were recorded during the annealing

148 phase of each cycle. On each plate, a standard curve is generated from $250 \mathrm{pg}$ to $100 \mathrm{ng}$, and

149 when the $R^{2}$ value was $>0.99$, the cycle threshold $(\mathrm{Ct})$ values were accepted. The GAPDH

150 housekeeping gene was used for normalization of target gene expression.

151 Analysis of APN secretion. Quantification of APN protein levels in cell culture supernatants was

152 determined with mouse APN ELISA kit (Phoenix Pharmaceuticals, Inc., Belmont, CA) per

153 manufacturer's instructions.

154 Western blot analysis: Cardiomyocyte lysates were separated by SDS/PAGE (12\% acrylamide

155 gel) and transferred to polyvinylidene difluoride membranes. Nonspecific binding sites were

156 blocked by $5 \%(\mathrm{wt} / \mathrm{vol})$ dried skimmed milk in TBS detergent $(0.1 \%$ Tween 20$)$. Membranes

157 were then probed with specific primary antibodies followed by washing ( $3 \mathrm{x}$ for $15 \mathrm{~min}$ ) with

158 TBS detergent $(0.1 \%$ Tween 20$)$. The membranes were then incubated with either goat anti-

159 mouse Ig or goat anti-rabbit Ig conjugated with horseradish peroxidase (1:10,000; Bio-Rad) for 1

$160 \mathrm{~h}$ and washed (three times for $15 \mathrm{~min}$ ) with TBS detergent ( $0.1 \%$ Tween 20$)$. Immunoreactivity

161 was visualized by using a Supersignal Chemiluminescence detection kit (Pierce, Rockford, Ill).

162 Immunoblotting was visualized with a Kodak Image Station 400 (Rochester, NY), and the blot

163 densities were analyzed with Kodak 1-dimensional software. 
164 Measurement of caspase-3 activity: Apoptotic cell death was determined by caspase-3 activation, 165 via a fluorometric kit (R\&D System, Minneapolis, MN). Briefly, cardiomyocytes were harvested 166 using caspase lysis buffers (50mM HEPES PH 7.4, 0.1\% Chaps, 5mM DTT, $0.1 \mathrm{mM}$ EDTA, $1670.1 \%$ Triton-X100). $50 \mu \mathrm{g}$ cell lysate samples were used to perform the fluorometric assay per 168 manufacturer's instructions. The fluorescence emission of the 7-amino-4-trifluoromethyl169 coumarin (AFC), released on proteolytic cleavage of the fluorogenic substrate DEVD-AFC by 170 active caspase-3, was measured using Biotek FL600 microplate fluorescence reader (excitation 171 wavelength, $400 \mathrm{~nm}$; emission wavelength, $505 \mathrm{~nm}$ ). Caspase-3 activity was expressed as nmol $172 A F C / \mathrm{h} / \mathrm{mg}$ protein.

173 Lactate dehydrogenase ( $L D H)$ release assay: At the end of observation period, conditioned

174 media was collected, and cells were lysed. LDH activity in conditioned media and cell lysates 175 was determined spectrophotometrically (SpectraMax-Plus microplate spectrophotometer, 176 Molecular Devices, Sunnyvale, CA). The percentage of LDH release was calculated as follows: $177(\mathrm{~A}-\mathrm{B}) /(\mathrm{C}-\mathrm{B}) \times 100$, where $\mathrm{A}=\mathrm{LDH}$ activity in conditioned media; $\mathrm{B}=\mathrm{LDH}$ activity in culture 178 media (without cells); $\mathrm{C}=\mathrm{LDH}$ activity in cell lysates.

179 Quantification of cellular nitrotyrosine content: Cardiomyocyte nitrotyrosine content, an index 180 of nitric oxide (NO) inactivation by superoxide and oxidative/nitrative stress, was determined by 181 a nitrotyrosine ELISA kit (Cell Sciences, Canton, MA) per manufacturer's instructions.

182 Statistical Analysis: All values in the text and figures are presented as means \pm SEM of $n$ 183 independent experiments. All data (except Western blot density) were subjected to ANOVA 184 followed by Bonferroni correction for post-hoc $t$ test. Western blot densities were analyzed with 185 the Kruskal-Wallis test followed by Dunn's post-hoc test. Probabilities of 0.05 or less were 186 considered to be statistically significant. 
188 Lack of APN, but not its receptors, significantly increased SI/R injury: Cardiomyocytes from

189 adult male WT, APN-KO, AdipoR1-KD, and AdipoR2-KO mice were subjected to sham SI/R

190 culture, or $3 \mathrm{~h}$ SI plus $12 \mathrm{~h}$ reperfusion. Cellular injury was determined by LDH release and

191 caspase-3 activation. As illustrated in Figure 1, SI/R caused significant LDH release and

192 caspase-3 activation in all groups studied (**P $<0.01$ vs. respective sham control). Lack of APN

193 (APN-KO) significantly increased SI/R injury, as evidenced by higher percentage of LDH

194 release (Figure 1A, second group, ${ }^{\#} \mathrm{P}<0.05$ vs. WT) and greater caspase-3 activity (Figure 1B,

195 second group, ${ }^{\#} \mathrm{P}<0.01$ vs. WT). No significant difference in SI/R injury was observed between

196 WT and APN receptor deficient cardiomyocytes $(\mathrm{P}>0.05)$, although LDH release and caspase-3

197 activation were slightly higher in cardiomyocytes from AdipoR1-KD/AdipoR2-KO mice. These

198 results indicate that the basal level of APN produced by adult cardiomyocytes is biologically

199 active in protecting cardiomyocytes against SI/R injury.

200 A peroxisome proliferator-activated receptor- $\gamma(P P A R \gamma)$ agonist regulates cardiomyocyte APN

201 and its receptors' expression: In order to better understand the biological function of locally

202 produced APN, cardiomyocytes were treated with rosiglitazone (RSG), a PPAR $\gamma$ agonist known

203 to stimulate APN expression and secretion in adipocytes. Based on our pilot time-course

204 observation, APN mRNA/protein expression, APN protein secretion, AdipoR1, and AdipoR2

205 protein expression were determined $6 \mathrm{~h}$ after RSG treatment. As summarized in Figure 2, 206 treatment of WT cardiomyocytes with RSG caused a 1.9-fold increase in APN mRNA

207 expression, 2.7-fold increase in APN protein expression, and 1.6-fold increase in APN level in 208 culture medium (P values for all <0.01). These results demonstrated that cardiomyocyte APN 209 expression (at both mRNA and protein level) and secretion are regulated by the PPAR $\gamma$ system. 
The adult cardiomyocytes used in this study has no adipocyte contamination as

211 determined by Oil-red O staining (data not shown). To further enhance our confidence that

212 PPAR $\gamma$-stimulated APN production is of cardiomyocytes origin, H9C2 cells (rat cardiac cell line)

213 were treated with vehicle or RSG for 6 hours. Comparable as that seen in adult cardiomyocytes,

214 treatment of $\mathrm{H} 9 \mathrm{C} 2$ cells also significantly increased APN mRNA expression (1.78 \pm 0.24 over

215 vehicle group, $\mathrm{P}<0.01)$, APN protein expression $(2.51 \pm 0.31$ over vehicle group, $\mathrm{P}<0.01)$ and

216 APN level in culture medium $(1.71 \pm 0.22$ over vehicle group, $\mathrm{P}<0.01)$.

217 To gain more insight into PPAR $\gamma$ regulation of cardiac APN systems, additional

218 experiments were performed to determine the effect of RSG on APN, AdipoR1, and AdipoR2

219 expression in WT cardiomyocytes and gene-manipulated cardiomyocytes. As expected, APN

220 expression was not detected in APN-KO cardiomyocytes; response to RSG was completely lost

221 in these cells (Figure 3, second group). The AdipoR1-KD or AdipoR2-KO condition alone

222 neither altered basal cardiomyocyte APN production, nor their response to RSG (Figure 3, third

223 and fourth group). However, basal APN expression was significantly increased in AdipoR-

$224 \mathrm{KD} /$ AdipoR2-KO cardiomyocytes $(\mathrm{P}<0.05)$, and the RSG response was significantly potentiated

225 in these cells (Figure 3, last group. $\mathrm{P}<0.01$ ).

226 RSG treatment significantly increased AdipoR1 expression in WT cardiomyocytes

227 (Figure 4, first group), and lack of APN neither altered basal AdipoR1 expression, nor its

228 response to RSG (Figure 4, second group). Our method of intra-myocardial siRNA delivery was

229 highly successful. Basal AdipoR1 expression was markedly inhibited ( $>80 \%)$ when siRNA

230 against AdipoR1 was injected, and upregulatory response to RSG was completely abolished in

231 these cells (Figure 4, third group). Basal and RSG-stimulated AdipoR1 expression was unaltered

232 in AdipoR2-KO cardiomyocytes (Figure 4, fourth group). Similar to AdipoR1, AdipoR2 
233 expression was also upregulated by RSG. Lack of APN or AdipoR1 neither altered basal

234 AdipoR2 expression, nor its upregulation by RSG (Figure 5).

235 Role of cardiomyocyte-derived APN in RSG cardioprotection and its receptor involvement:

236 Having demonstrated that the lack of cardiomyocyte-derived basal APN production exaggerated

237 SI/R injury (Figure 1), and that RSG upregulated the expression of APN and its receptors

238 (Figures 2-5), we further determined whether cardiomyocyte-derived APN is also biologically

239 active in mediating RSG's cardioprotective effects. Cardiomyocytes from WT, APN-KO,

240 AdipoR1-KD, AdipoR2-KO, and AdipoR1-KD/AdipoR2-KO mice were treated with vehicle or

241 RSG for $6 \mathrm{~h}$ as described above, followed by $3 \mathrm{~h}$ simulated ischemia and $12 \mathrm{~h}$ reperfusion. In

242 WT cardiomyocytes, RSG pre-treatment significantly reduced SI/R injury, as evidenced by

243 significantly reduced LDH release and attenuated caspase-3 activation (Figure 6, first group). In

244 contrast, no significant cardioprotective effects of RSG were observed in cardiomyocytes from

245 APN-KO mice (Figure 6, second group), indicating that in vitro cardioprotective effects of RSG

246 are largely mediated by locally produced APN. Moreover, the cardioprotective effects of RSG

247 was markedly reduced, but not completely lost, in cardiomyocytes isolated from AdipoR1-KD

248 mice (Figure 6, third group). Although AdipoR2 is expressed in cardiomyocytes, complete

249 knockout of adiponectin receptor 2 had less profound effect than adiponectin receptor 1 knock-

250 down on RSG cardioprotection in this in vitro simulated ischemia/reperfusion model (Figure 6,

251 fourth group). Finally, cardiomyocytes isolated from AdipoR1-KD/AdipoR2-KO mice

252 responded to RSG similarly as AdipoR1-KD cardiomyocytes. These results demonstrated that

253 increased cardiomyocyte APN production as a result of RSG treatment is largely responsible for

254 the cardioprotective effects of RSG, and such effects are primarily mediated by AdipoR1

255 activation. 
256 Role of APN in RSG anti-nitrative signaling and its receptor involvement: Our previous studies

257 have demonstrated that in vivo systemic APN cardioprotective effects are largely mediated by its 258 anti-oxidative/anti-nitrative effect(26). To further investigate the intracellular mechanisms 259 responsible for cardiomyocyte-derived APN cardioprotection, the effect of manipulating APN or 260 APN receptors on RSG anti-oxidative/anti-nitrative effect was assessed. In cardiomyocytes 261 isolated from WT mice, SI/R caused a greater than 3-fold increase in cellular nitrotyrosine 262 content, which was markedly reduced by RSG pre-treatment (Figure 7, first group). SI/R263 induced cardiomyocyte nitrotyrosine overproduction was significantly further increased in APN264 KO cardiomyocytes (Figure 7, second group). The anti-oxidant/anti-nitrative effect of RSG was 265 lost in these cells, with a highly significant difference observed between RSG-treated WT versus 266 RSG-treated APN-KO cardiomyocytes $(\mathrm{P}<0.01)$. Neither AdipoR1-KD nor AdipoR2-KO had 267 significant effect on SI/R-induced nitrotyrosine overproduction (Figure 7, third to fifth group). 268 However, the anti-oxidative/anti-nitrative effect of RSG was abrogated in AdipoR1-KD and 269 AdipoR1-KD/AdipoR2-KO cardiomyocytes (Figure 7). 


\section{$\underline{\text { Discussion }}$}

APN is a protein hormone that modulates a number of metabolic processes, including

276 glucose regulation and fatty acid catabolism(2;3). The primary sequence of the polypeptide

277 contains a signal sequence (cleaved in the mature protein) and a non-conserved N-terminal

278 domain, followed by 22 collagen repeats, and a C-terminal globular domain that has structural

279 similarities to $\mathrm{TNF} \alpha(13)$. Like all collagen domain-containing proteins, full length APN

280 spontaneously forms a homotrimer basic unit. The homotrimer self-associates through conserved

281 N-terminal cysteine residues to form disulfide-linked hexamers, which further assemble into

282 high-molecular weight forms consisting of multiple oligomers of the basic trimer unit. A

283 proteolytic cleavage product of APN containing the globular C-terminal domain (gAPN) has

284 been postulated to exist in vivo(6). A recent study has shown that the cleavage of full length APN

285 by leukocyte elastase (secreted by activated monocytes and/or neutrophils) could be responsible

286 for gAPN generation(27).

287 For many years, APN was believed to be produced and secreted exclusively by

288 adipocytes. As such, although APN has been well-accepted as a potent cardioprotective molecule,

289 clinical and experimental studies have been completely focused on identifying the relationship

290 between systemic APN and cardiovascular injury( $1 ; 7 ; 12 ; 16 ; 23 ; 24)$. In 2005, Pineiro and

291 colleagues reported that human and murine cardiomyocytes are capable of producing APN(22),

292 an observation subsequently confirmed by other investigators $(4 ; 11)$. However, a critical

293 investigation concerning the biological activity and physiologic/pathologic relevance of

294 cardiomyocyte-derived APN remained unexplored.

295 Using two different approaches in our study, we have obtained clear evidence that 296 cardiomyocyte-derived APN is both biologically active and pathologically relevant. First, we 
297 have demonstrated that simulated ischemia/reperfusion injury is significantly potentiated in 298 cardiomyocytes lacking APN, indicating that APN is constantly produced by adult 299 cardiomyocytes, and that this basal level of locally produced APN is indeed biologically active 300 in its protection of cardiomyocytes from ischemia/reperfusion injury. Second, we have 301 demonstrated that cardiomyocyte APN production is upregulated by the PPAR $\gamma$ system, and 302 more importantly, the cardioprotective effect of RSG was completely lost in cardiomyocytes 303 lacking APN. To our knowledge, this is the first direct evidence demonstrating that 304 cardiomyocyte-derived APN is the most important molecule mediating the cardioprotective 305 actions of RSG- clearly making APN of local origin a key player in pharmacologic therapeutic 306 intervention success.

307 Currently, it is generally accepted that APN regulates cellular function by 308 binding/activating its specific receptors. Two types of APN receptor have been cloned(28). 309 Whereas AdipoR1 is abundantly expressed in muscular cells, AdipoR2 is predominantly 310 expressed in the liver. They belong to a new family of membrane receptors predicted to contain 311 seven transmembrane domains, but are structurally and topologically distinct from G-protein 312 coupled receptors. APN binds to the C-terminal extracellular domain of AdipoR, whereas the N313 terminal cytoplasmic domain interacts with an adaptor protein, APPL1(18). In addition to these

314 two receptors, T-cadherin has been proposed to be a receptor for hexameric and high molecular 315 weight forms of APN(14). However, the biologic function of APN/T-cadherin binding remains 316 unclear, because T-cadherin lacks an intracellular domain.

317 The current study raised several interesting observations concerning APN receptors and 318 their involvement in locally produced APN-mediated cardioprotection. First, the absence of APN 319 did not alter basal expression of the APN receptor, and it did not affect RSG-induced receptor 
320 upregulation. However, although the absence of one of the two APN receptor types did not 321 affect APN expression, deficiency of both APN receptor types significantly upregulated APN 322 basal expression, and potentiated RSG response. The pathological significance of this interesting 323 phenomenon warrants more direct investigation. Second, APN deficiency had more significant 324 impact on cardiomyocyte response to ischemia/reperfusion than its receptors had. Specifically, 325 under basal condition, cardiomyocytes lacking APN, but not APN receptors, had significantly 326 higher LDH release and caspase-3 activation after SI/R (Figure 1). Under RSG stimulatory 327 condition, the RSG cardioprotective effect was completely lost in cardiomyocytes lacking APN, 328 but not in cardiomyocytes lacking APN receptors. Precise mechanisms responsible for this 329 discrepancy can not be addressed in the current study. However, several possibilities exist. In the 330 current study, AdipoR1 siRNA caused approximately $80 \%$ reduction of AdipoR1 expression. 331 The remaining AdipoR1 might be responsible for partial protection in AdipoR-KD 332 cardiomyocytes. Alternatively, other APN receptors, such as T-cadherin, might be present in 333 adult cardiomyocytes and be able to translate APN cardioprotective signaling in AdipoR1$334 \mathrm{KD} /$ AdipoR2-KO cardiomyocytes. Finally, cardiomyocyte-derived APN may participate in 335 cardioprotective signaling via intracellular protein-protein interaction. Additional experiments 336 directly investigating these possibilities are currently under investigation.

Third, although AdipoR1 and AdipoR2 are known to be constitutively expressed in adult 338 cardiomyocytes, each receptor's relative contribution to APN cardioprotective signaling remains 339 unclear. As illustrated in Figures 6 and 7, manipulation of AdipoR1 had greater impact on RSG's 340 cardioprotective and anti-oxidative/anti-nitrative actions than alteration of AdipoR2, despite the 341 complete knockout of AdipoR2, whereas AdipoR1 was only partially (knock-down) lost in 342 cardiomyocytes studied. At least two possibilities may explain this discrepancy. First, it is 
343 possible that the cardioprotective effects of locally produced APN are primarily mediated by 344 AdipoR1, since cardiomyocytes are AdipoR1 dominant. Second, cardiomyocyte-derived APN 345 may contain more low-molecular weight APN, which has high affinity to AdipoR1. Additional 346 experiments are currently undertaken to directly address these possibilities.

347 In summary, our studies demonstrated for the first time that locally-produced APN by 348 cardiomyocytes is biologically active, pathologically significant, and pharmacologically 349 important. This locally-produced APN protects cardiomyocyte against ischemia/reperfusion 350 injury primarily via paracrine/autocrine activation of AdipoR1. Furthermore, similar to 351 circulatory APN, locally-produced APN protects against cardiomyocyte injury through reduction 352 of ischemia/reperfusion-induced oxidative/nitrative stress. 
1. Behrends M, Schulz R, Post H, Alexandrov A, Belosjorow S, Michel MC and Heusch

G. Inconsistent relation of MAPK activation to infarct size reduction by ischemic preconditioning in pigs. Am J Physiol 279: 1111-1119, 2000.

2. Berg AH, Combs TP and Scherer PE. ACRP30/adiponectin: an adipokine regulating glucose and lipid metabolism. Trends Endocrinol Metab 13: 84-89, 2002.

3. Chandran M, Phillips SA, Ciaraldi T and Henry RR. Adiponectin: more than just another fat cell hormone? Diabetes Care 26: 2442-2450, 2003.

4. Ding G, Qin Q, He N, Francis-David SC, Hou J, Liu J, Ricks E and Yang Q. Adiponectin and its receptors are expressed in adult ventricular cardiomyocytes and upregulated by activation of peroxisome proliferator-activated receptor gamma. $J$ Mol Cell Cardiol 43: 73-84, 2007.

5. Forrat R, Sebbag L, Wiernsperger N, Guidollet J, Renaud S and De Lorgeril M. Acute myocardial infarction in dogs with experimental diabetes. Cardiovasc Res 27: 19081912, 1993. and Lodish HF. Proteolytic cleavage product of 30-kDa adipocyte complement-related protein increases fatty acid oxidation in muscle and causes weight loss in mice. PNAS 98: 2005-2010, 2001. 
7. Frystyk J, Berne C, Berglund L, Jensevik K, Flyvbjerg A and Zethelius B. Serum adiponectin is a predictor of coronary heart disease: a population-based 10-year follow-up study in elderly men. J Clin Endocrinol Metab 92: 571-576, 2007.

8. Gao F, Gao E, Yue TL, Ohlstein EH, Lopez BL, Christopher TA and Ma XL. Nitric oxide mediates the antiapoptotic effect of insulin in myocardial ischemia-reperfusion: the roles of PI3-kinase, Akt, and endothelial nitric oxide synthase phosphorylation. Circulation 105: 1497-1502, 2002.

9. Goldstein BJ and Scalia R. Adipokines and vascular disease in diabetes. Curr Diab Rep 7: 25-33, 2007.

10. Goldstein BJ, Scalia RG and Ma XL. Protective vascular and myocardial effects of adiponectin. Nat Clin Pract Cardiovasc Med 6: 27-35, 2009.

11. Guo Z, Xia Z, Yuen VG and McNeill JH. Cardiac expression of adiponectin and its receptors in streptozotocin-induced diabetic rats. Metabolism 56: 1363-1371, 2007.

12. Hotta K, Funahashi T, Arita Y, Takahashi M, Matsuda M, Okamoto Y, Iwahashi H, Kuriyama H, Ouchi N, Maeda K, Nishida M, Kihara S, Sakai N, Nakajima T, Hasegawa K, Muraguchi M, Ohmoto Y, Nakamura T, Yamashita S, Hanafusa T and Matsuzawa Y. Plasma concentrations of a novel, adipose-specific protein, adiponectin, in type 2 diabetic patients. Arterioscler Thromb Vasc Biol 20: 1595-1599, 2000.

13. Hug C and Lodish HF. The role of the adipocyte hormone adiponectin in cardiovascular disease. Curr Opin Pharmacol 5: 129-134, 2005. 
14. Hug C, Wang J, Ahmad NS, Bogan JS, Tsao TS and Lodish HF. T-cadherin is a receptor for hexameric and high-molecular-weight forms of Acrp30/adiponectin. Proc Natl Acad Sci U S A 101: 10308-10313, 2004.

15. Jagasia D and McNulty PH. Diabetes mellitus and heart failure. Congest Heart Fail 9: 133-139, 2003.

16. Kumada M, Kihara S, Sumitsuji S, Kawamoto T, Matsumoto S, Ouchi N, Arita Y, Okamoto Y, Shimomura I, Hiraoka H, Nakamura T, Funahashi T and Matsuzawa Y. Association of hypoadiponectinemia with coronary artery disease in men. Arterioscler Thromb Vasc Biol 23: 85-89, 2003.

\section{Liu Y, Chewchuk S, Lavigne C, Brule S, Pilon G, Houde V, Xu A, Marette A and} Sweeney G. Functional significance of skeletal muscle adiponectin production, changes in animal models of obesity and diabetes, and regulation by rosiglitazone treatment. Am J Physiol Endocrinol Metab 297: E657-E664, 2009.

\section{Mao X, Kikani CK, Riojas RA, Langlais P, Wang L, Ramos FJ, Fang Q, Christ-} Roberts CY, Hong JY, Kim RY, Liu F and Dong LQ. APPL1 binds to adiponectin receptors and mediates adiponectin signalling and function. Nat Cell Biol 8: 518-523, 2006.

19. Marfella R, D'Amico M, Di Filippo C, Piegari E, Nappo F, Esposito K, Berrino L, Rossi F and Giugliano D. Myocardial infarction in diabetic rats: role of hyperglycaemia on infarct size and early expression of hypoxia-inducible factor 1. Diabetologia 45: 1172$1181,2002$. 
20. Namiki A, Brogi E, Kearney M, Kim EA, Wu T, Couffinhal T, Varticovski L and Isner JM. Hypoxia induces vascular endothelial growth factor in cultured human endothelial cells. J Biol Chem 270: 31189-31195, 1995.

21. Ouchi N, Shibata R and Walsh K. Cardioprotection by adiponectin. Trends CardiovasC Med 16: 141-146, 2006.

22. Pineiro R, Iglesias MJ, Gallego R, Raghay K, Eiras S, Rubio J, Dieguez C, Gualillo O, Gonzalez-Juanatey JR and Lago F. Adiponectin is synthesized and secreted by human and murine cardiomyocytes. FEBS Lett 579: 5163-5169, 2005.

23. Pischon T, Girman CJ, Hotamisligil GS, Rifai N, Hu FB and Rimm EB. Plasma adiponectin levels and risk of myocardial infarction in men. JAMA 291: 1730-1737, 2004.

24. Shibata R, Numaguchi Y, Matsushita K, Sone T, Kubota R, Ohashi T, Ishii M, Kihara S, Walsh K, Ouchi N and Murohara T. Usefulness of adiponectin to predict myocardial salvage following successful reperfusion in patients with acute myocardial infarction. Am J Cardiol 101: 1712-1715, 2008.

25. Shinoda Y, Yamaguchi M, Ogata N, Akune T, Kubota N, Yamauchi T, Terauchi Y, Kadowaki T, Takeuchi Y, Fukumoto S, Ikeda T, Hoshi K, Chung UI, Nakamura K and Kawaguchi $\mathbf{H}$. Regulation of bone formation by adiponectin through autocrine/paracrine and endocrine pathways. J Cell Biochem 99: 196-208, 2006.

26. Tao L, Gao E, Jiao X, Yuan Y, Li S, Christopher TA, Lopez BL, Koch W, Chan L, Goldstein BJ and Ma XL. Adiponectin cardioprotection after myocardial 
ischemia/reperfusion involves the reduction of oxidative/nitrative stress. Circulation 115: 1408-1416, 2007.

27. Waki H, Yamauchi T, Kamon J, Kita S, Ito Y, Hada Y, Uchida S, Tsuchida A,

Takekawa S and Kadowaki T. Generation of globular fragment of adiponectin by leukocyte elastase secreted by monocytic cell line THP-1. Endocrinology 146: 790-796, 2005.

28. Yamauchi T, Kamon J, Ito Y, Tsuchida A, Yokomizo T, Kita S, Sugiyama T,

29. Zhu M, Miura J, Lu LX, Bernier M, DeCabo R, Lane MA, Roth GS and Ingram DK. sensitization. Exp Gerontol 39: 1049-1059, 2004. 


\section{$453 \quad$ Funding Sources}

454

455 This research was supported by the following grants: NIH 2R01HL-63828, American Diabetes

456 Association Research Award 7-08-RA-98, American Heart Association Grant-in-Aid 0855554D

457 (to X.L.M.), and the Emergency Medicine Foundation Career Development Grant (to W.B.L.).

460 Conflict of interest: none declared 
464 Figure 1. Simulated ischemia/reperfusion injury assessed by LDH release (A) and caspase-3 activation (B). Assays were performed at the end of $3 \mathrm{~h}$ of SI and $12 \mathrm{~h}$ of reperfusion (SI/R) or $15 \mathrm{~h}$ of normal culture (Sham). ${ }^{* *} \mathrm{P}<0.01$ vs. sham $\mathrm{SI} / \mathrm{R} ;{ }^{*} \mathrm{P}<0.05,{ }^{\# \#} \mathrm{P}<0.01$ vs. WT cardiomyocytes with SI/R. N=14-16 wells/group with cardiomyocytes isolated from 6 to 8 mice.

Figure 3. Effect of RSG on APN protein expression in cardiomyocytes isolated from WT or

Figure 2. Effect of rosiglitazone (RSG) on adiponectin (APN) mRNA expression (A), APN protein expression (B) and APN secretion (C) in C57BL/6 cardiomyocytes. Assays were performed $6 \mathrm{~h}$ after vehicle or RSG treatment. $* * \mathrm{P}<0.01$ vs. vehicle; $\mathrm{N}=14-16$ wells/group with cardiomyocytes isolated from 6 to 8 mice. gene manipulated mice. Assays were performed $6 \mathrm{~h}$ after vehicle or RSG treatment. ${ }^{*} \mathrm{P}<0.05,{ }^{*} \mathrm{P}<0.01$ vs. vehicle in the same group; ${ }^{\#} \mathrm{P}<0.05,{ }^{\# \#} \mathrm{P}<0.01$ vs. WT cardiomyocytes with the same treatment. $\mathrm{N}=14-16$ wells/group with cardiomyocytes isolated from 6 to 8 mice.

Figure 4. Effect of RSG on adiponectin receptor 1 (AdipoR1) expression in cardiomyocytes isolated from WT or gene manipulated mice. Assays were performed $6 \mathrm{~h}$ after vehicle or RSG treatment. $* \mathrm{P}<0.05$ vs. vehicle in the same group; $\mathrm{N}=14-16$ wells/group with cardiomyocytes isolated from 6 to 8 mice. 
485

Figure 5. Effect of RSG on adiponectin receptor 2 (AdipoR2) expression in cardiomyocytes isolated from WT or gene manipulated mice. Assays were performed $6 \mathrm{~h}$ after vehicle or RSG treatment. $* \mathrm{P}<0.05$ vs. vehicle in the same group; $\mathrm{N}=14-16$ wells/group with cardiomyocytes isolated from 6 to 8 mice.

Figure 6. Effect of APN, AdipoR1 and AdipoR2 deficiency on RSG cardioprotection against SI/R injury. Cardiomyocytes were pretreated with vehicle or RSG for $6 \mathrm{~h}$ followed by $3 \mathrm{~h}$ of SI and $12 \mathrm{~h}$ of reperfusion. All data were normalized against mean value of their own vehicle group. ${ }^{*} \mathrm{P}<0.05,{ }^{*} \mathrm{P}<0.01$ vs. vehicle in the same group; ${ }^{\# \#} \mathrm{P}<0.01$ vs. WT cardiomyocytes with the same treatment. $\mathrm{N}=14-16$ wells/group with cardiomyocytes isolated from 6 to 8 mice.

Figure 7. Effect of APN, AdipoR1, and AdipoR2 deficiency on RSG mediated antioxidative/anti-nitrative effect in SI/R cardiomyocytes. Cardiomyocytes were pretreated with vehicle or RSG for $6 \mathrm{~h}$ followed by $3 \mathrm{~h}$ of SI and $12 \mathrm{~h}$ of reperfusion. All data were normalized against mean value of their own vehicle group. $* \mathrm{P}<0.05$, ${ }^{* *} \mathrm{P}<0.01$ vs. vehicle in the same group; ${ }^{\#} \mathrm{P}<0.05,{ }^{\# \#} \mathrm{P}<0.01$ vs. WT cardiomyocytes with the same treatment. N=14-16 wells/group with cardiomyocytes isolated from 6 to 8 mice. 

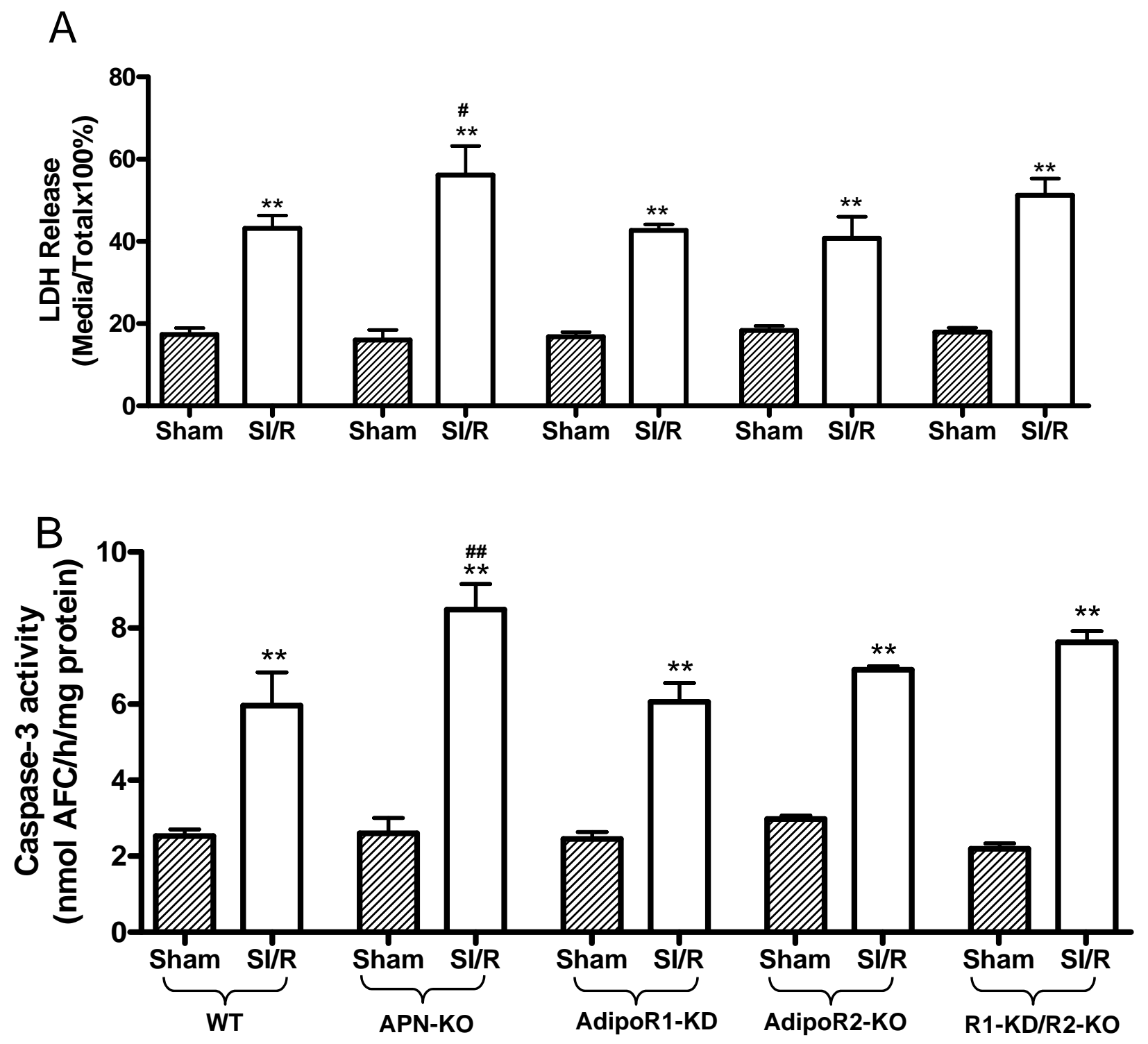

Figure 1 
A

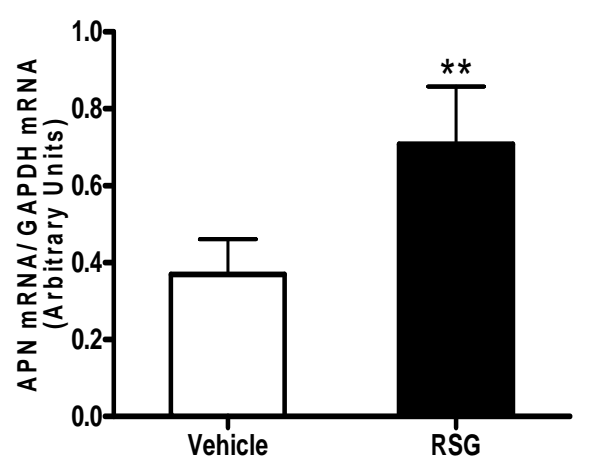

B

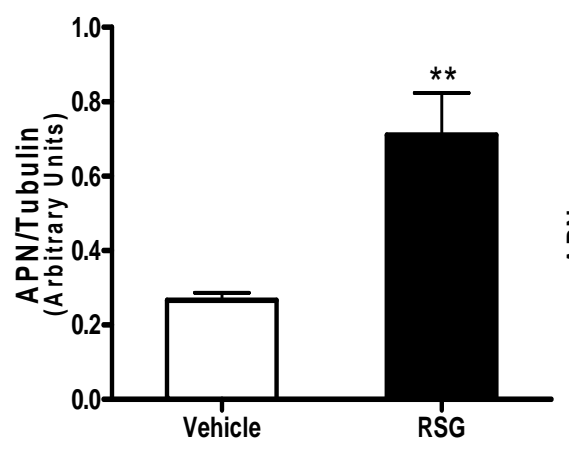

C

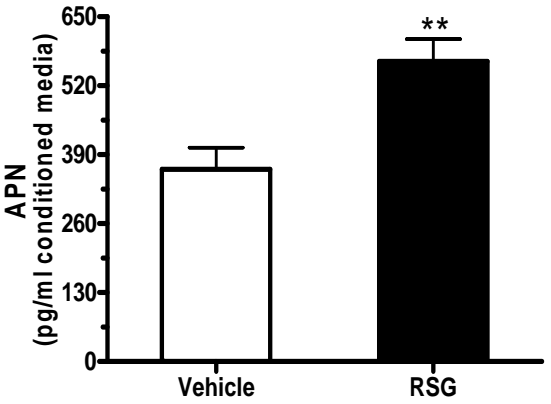

Figure 2 


\section{APN}

Tubulin

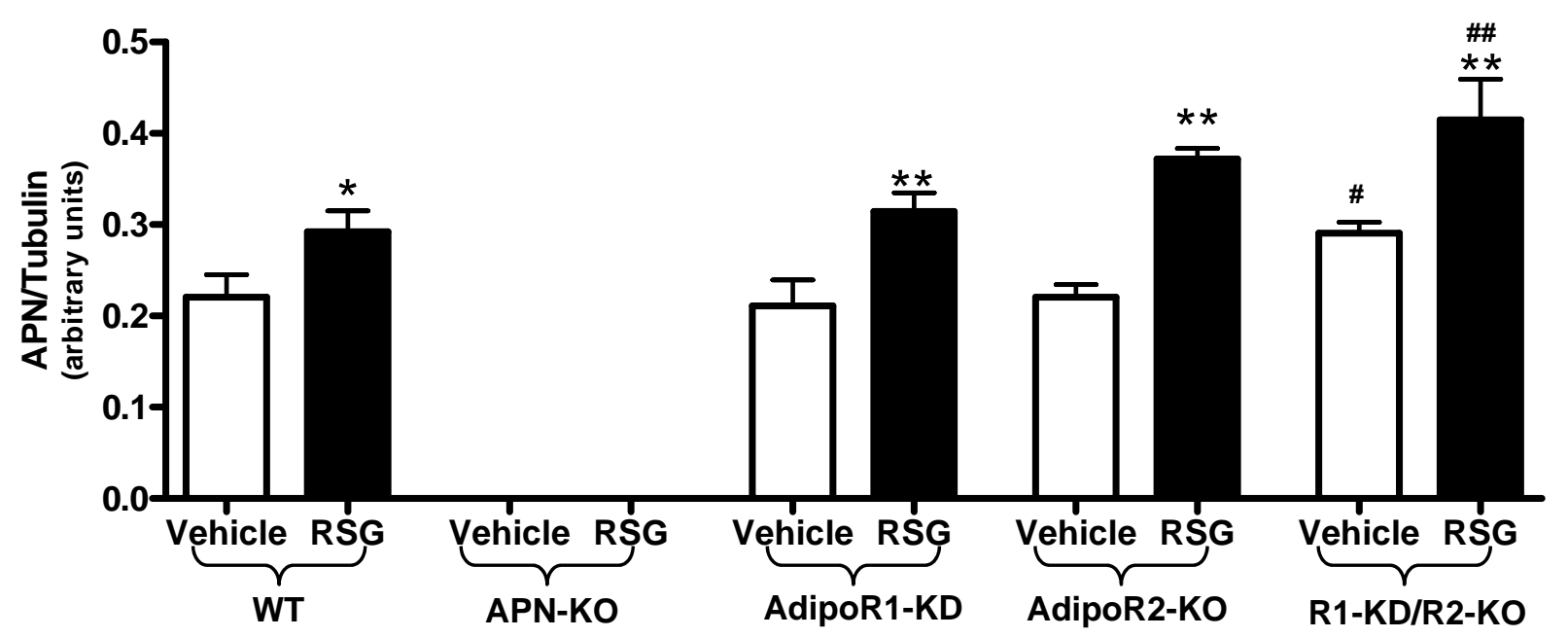

Figure 3 
AdipoR1

Tubulin

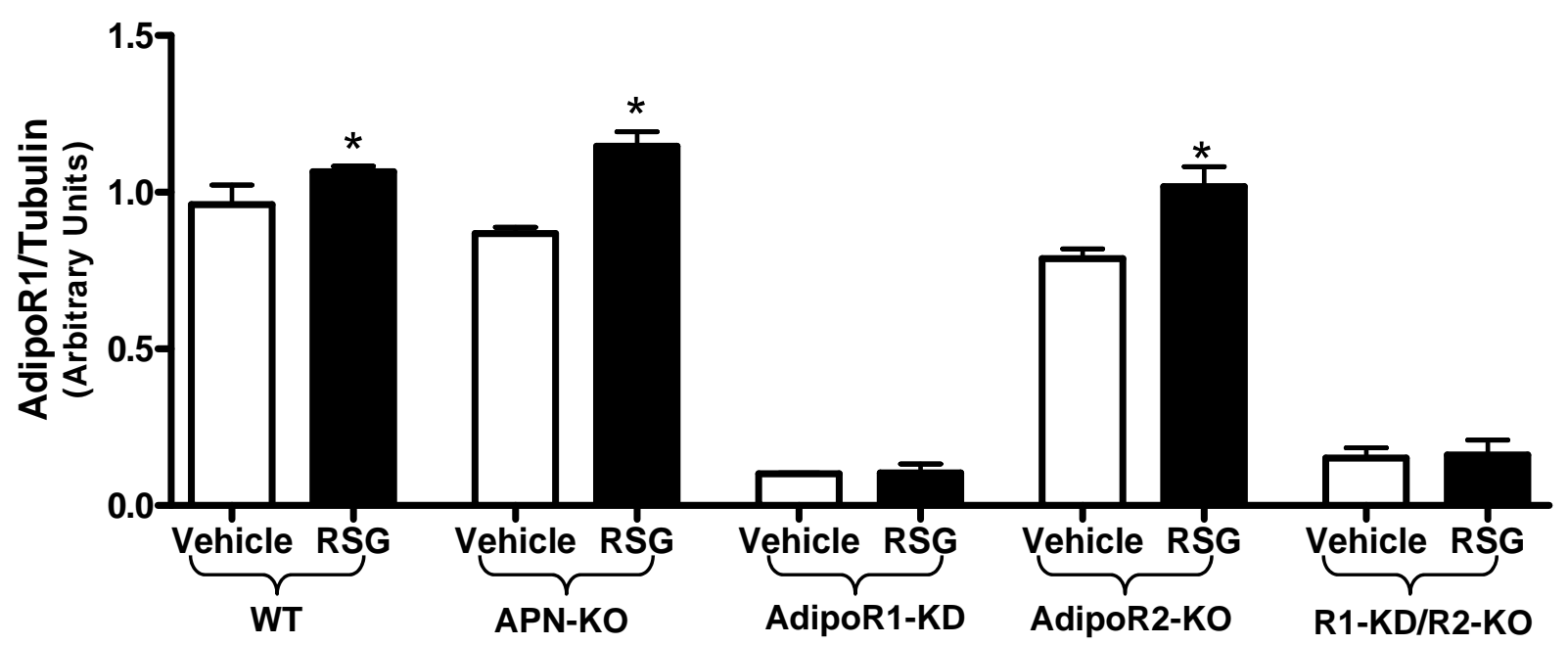

Figure 4 
AdipoR2

Tubulin

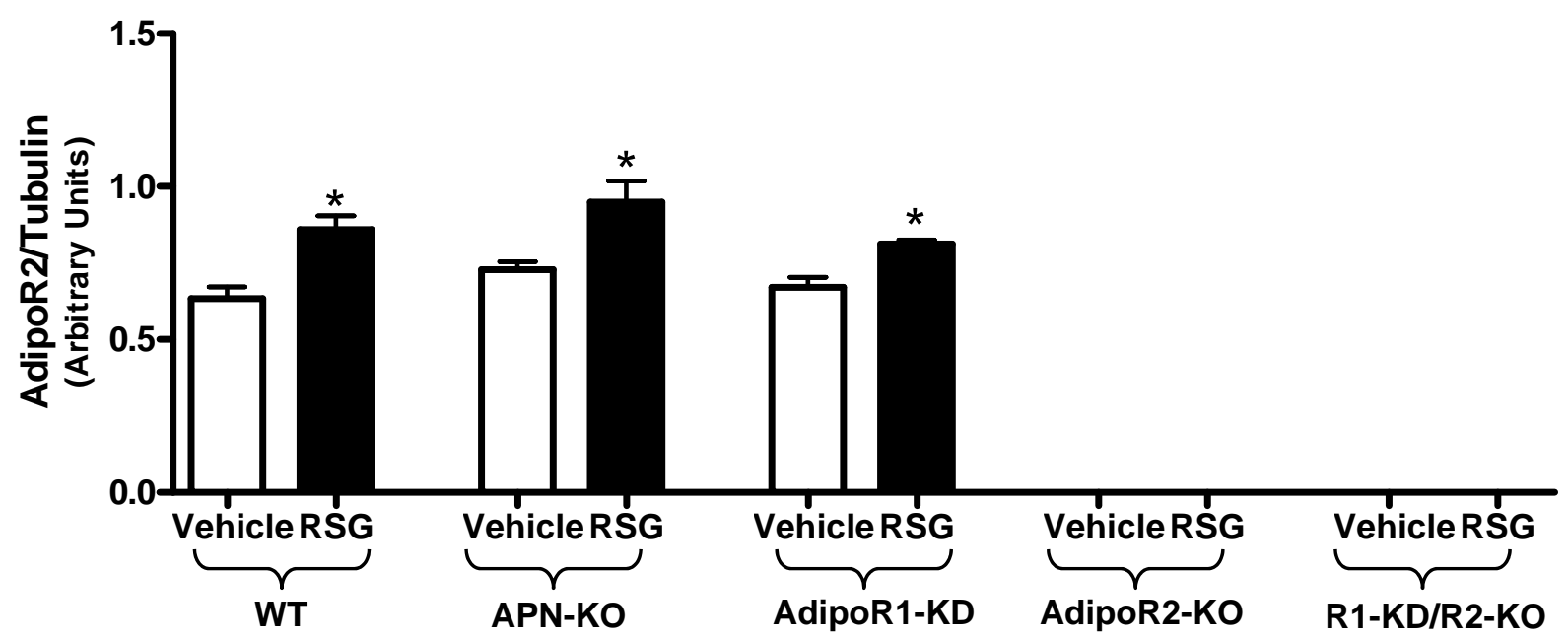

Figure 5 

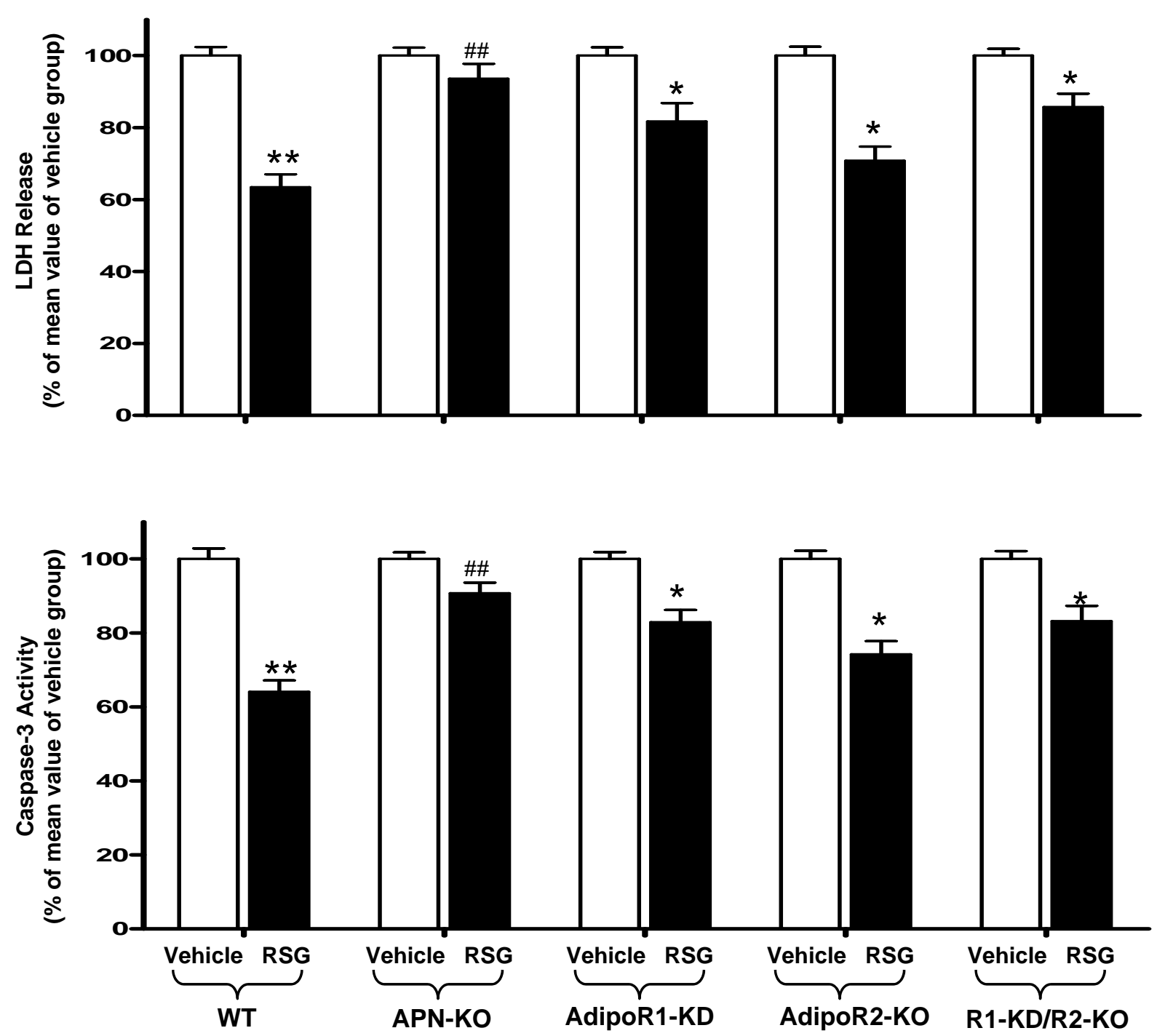

Figure 6 


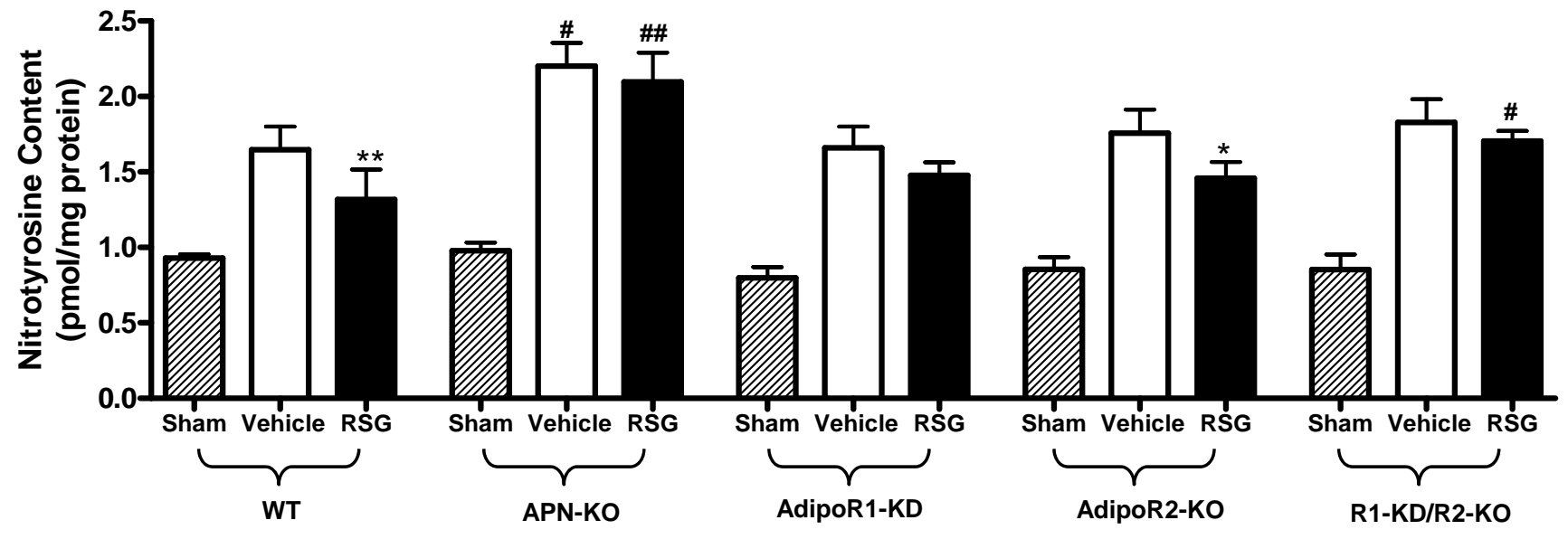

Figure 7 